ARQGA/1147

\title{
TUMOR SÓLIDO-CÍSTICO PSEUDOPAPILAR DO PÂNCREAS (TUMOR DE FRANTZ). Estudo de quatro casos
}

\author{
Gabriel Domingues COSTA-NETO, Enio Campos AMICO e Gabriel Izar Domingues COSTA
}

RESUMO - Racional - O tumor sólido pseudopapilar do pâncreas tem sido recentemente considerado menos raro que quando de sua descrição original por Frantz, em 1959. Novos casos têm sido descritos nos últimos anos despertando o interesse pela neoplasia. Objetivos - Estudar aspectos clínicos, histopatológicos, terapêuticos e de prognóstico em casuística uni-institucional. Pacientes e Métodos - Quatro casos com tumor de Frantz foram avaliados no período de dezembro de 2000 a fevereiro de 2003. Resultados - Três dos quatro casos eram de adolescente do sexo feminino. Sintomas dispépticos e massa palpável representaram a manifestação inicial. O local preferencial do tumor foi a cabeça pancreática (75\%) e o tratamento cirúrgico foi a enucleação (um caso), duodenopancreatectomia (dois casos) e a pancreatectomia corpocaudal (um caso). Em todos os casos foi possível a ressecção completa da lesão com margens livres. Em dois pacientes o exame microscópico revelou presença de invasão venosa. Foi realizado estudo imunoistoquímico em três casos. Com um seguimento médio de 15 meses, não havia sido descrita recurrência do tumor em nenhum dos casos. Conclusão - Com exceção da localização preferencial do tumor na cabeça pancreática (75\%), os demais achados clínicos, histopatológicos, imunoistoquímicos e terapêuticos foram compatíveis com publicações prévias. O pequeno seguimento pós-operatório impossibilita conclusões sobre o prognóstico dos pacientes estudados.

DESCRITORES - Neoplasias pancreáticas. Carcinoma papilar.

\section{INTRODUÇÃO}

O tumor sólido pseudopapilar do pâncreas (TSPP), também conhecido como tumor de Frantz, embora raro, é uma neoplasia que tem sido cada vez mais descrita na literatura ${ }^{(8)}$. É encontrado na maioria dos casos em adolescente do sexo feminino sendo considerado uma neoplasia com pequeno grau de malignidade, desde que passível de ressecção cirúrgica completa, o que é possível na maioria dos casos. O conhecimento dos aspectos epidemiológicos e clínicos desse tipo de tumor pode levar à suspeita do diagnóstico no pré-operatório, o que sem dúvida pode permitir abordagem cirúrgica mais radical que intencione a ressecção por completo de lesão.

Apresenta-se casuística uni-institucional, sendo discutidos aspectos clínicos, histopatológicos, imunoistoquímicos terapêuticos e de prognóstico.

\section{PACIENTES E MÉTODOS}

No período de dezembro de 2000 a fevereiro de 2003, quatro pacientes tratados na Santa Casa de Misericórdia de Presidente Prudente, SP, foram incluídos neste estudo por serem portadores de TSPP.

Três pacientes eram adolescentes do sexo feminino com variação de idade entre 17 e 26 anos e um único paciente do sexo masculino com 42 anos.
Em três casos a apresentação clínica era inespecífica, com sintomas de desconforto abdominal e dispepsia. Em apenas um caso (sexo masculino) a queixa inicial foi de massa abdominal palpável. Em nenhum deles foi observada perda de peso. O exame físico, com exceção deste último caso, apresentava-se sem alterações. Exames laboratoriais, incluindo enzimas hepáticas e amilase, foram normais.

O primeiro exame solicitado a todos os pacientes foi a ultra-sonografia de abdome, que revelou, sem exceção, a presença de massa pancreática, sendo este seguido em metade dos pacientes de tomografia de abdome e ressonância nuclear magnética na outra metade. Em três pacientes o tumor estava localizado na cabeça do pâncreas e em apenas um no corpo e cauda pancreática (Figuras 1, 2, 3).

Em um caso (sexo masculino) o paciente havia recebido previamente o diagnóstico de pseudocisto pancreático, tendo sido submetido a drenagem endoscópica da lesão. Como complicação, apresentou dor abdominal e queda dos níveis de hematócrito, sendo operado de urgência para controle do sangramento. O paciente evoluiu com aumento progressivo da lesão, optando-se por sua ressecção 4 meses depois, quando se diagnosticou TSPP por exame anatomopatológico.

Nos pacientes do sexo feminino, apenas no caso 1 o diagnóstico pré-operatório foi compatível com cisto pancreático; nas demais já se suspeitava de TSPP. 


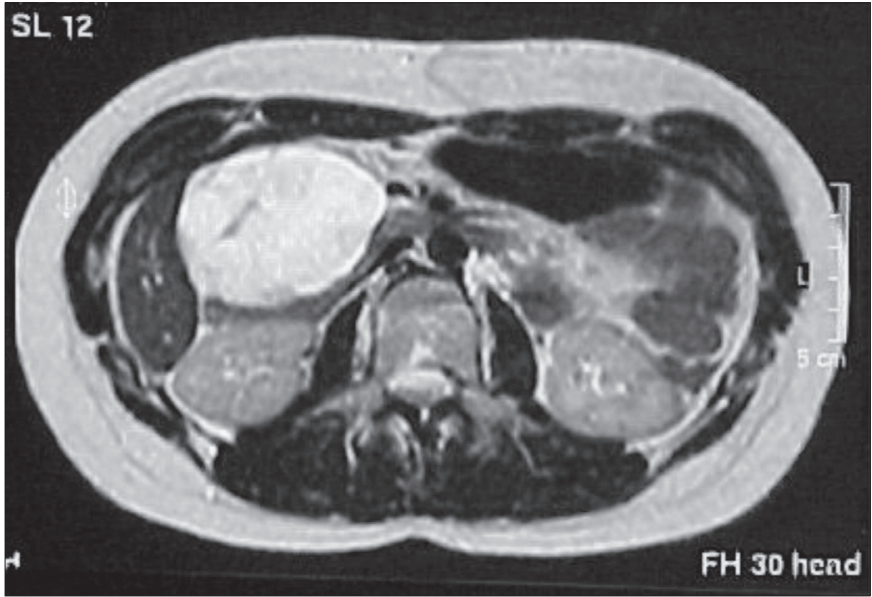

FIGURA 1 - Caso 1 - Imagem de RNM de abdome mostrando lesão em cabeça pancreática de $7,0 \mathrm{~cm}$ que se estende para espaço sub-hepático.

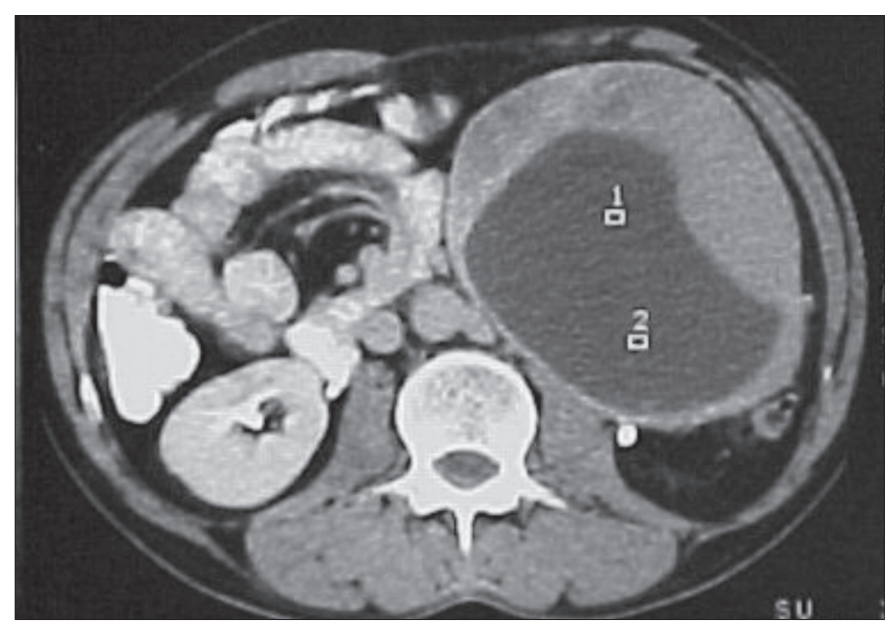

FIGURA 2 - Caso 2 - Imagem de CT de abdome com lesão cística-papilar de $15 \mathrm{~cm}$ em corpo e cauda pancreática.

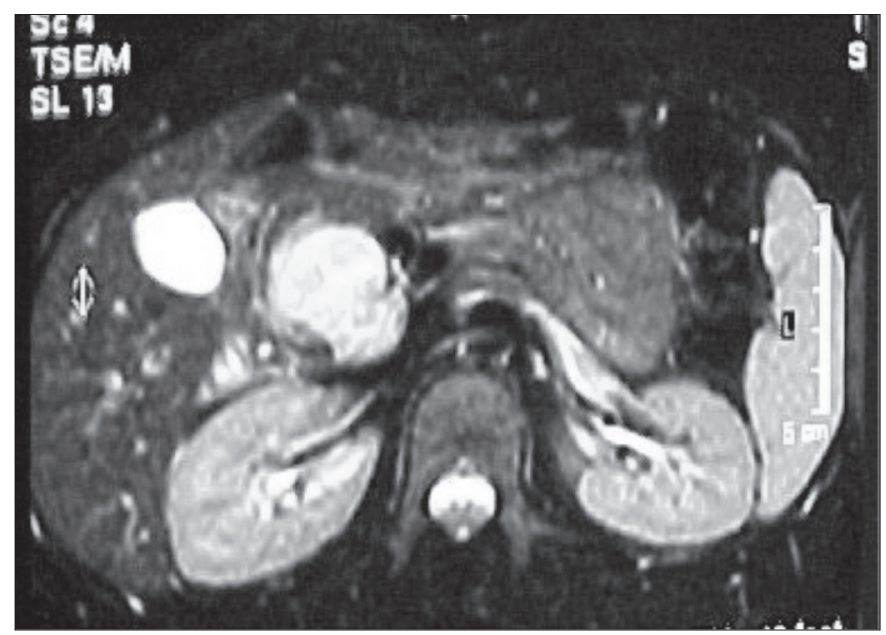

FIGURA 3 - Caso 3 - Imagem de RNM de abdome com lesão de $6,5 \mathrm{~cm}$ em topografia de cabeça pancreática.

\section{RESULTADOS}

O tratamento empregado encontra-se discriminado na Tabela 1. No caso 1, em virtude do tumor apresentar extensão extrapancreática, foi possível sua enucleação. No caso $2 \mathrm{em}$ que o tumor estava localizado em corpo e cauda pancreático e o paciente havia sido submetido previamente a drenagem endoscópica (já descrito anteriormente), foi identificada durante a cirurgia firme aderência do tumor ao rim esquerdo, sendo necessária a nefrectomia. No caso 4 houve lesão intra-operatória acidental da artéria mesentérica superior, sendo necessária a reconstrução com veia jugular externa. Esta paciente apresentou oclusão do enxerto sendo reoperada para implante de prótese de politetrafluoroetileno, evoluiu com quadro de sangramento digestivo alto e hepatite isquêmica, permanecendo por 29 dias na UTI, com alta após 35 dias de internação. Os demais casos evoluíram sem intercorrências. Não houve mortalidade na presente casuística.

TABELA 1 - Discriminação dos pacientes com relação às características do tumor e tratamento cirúrgico realizado

\begin{tabular}{|c|c|c|c|c|}
\hline Paciente & Idade & Sexo & Localização & Cirurgia \\
\hline Caso 1 & 17 & $\mathrm{~F}$ & Cabeça pancreática & Enucleação \\
\hline Caso 2 & 42 & M & $\begin{array}{l}\text { Cabeça e corpo } \\
\text { pancreático }\end{array}$ & $\begin{array}{l}\text { Pancreatectomia } \\
\text { corpo-caudal + esplenectomia } \\
\text { + nefrectomia esquerda }\end{array}$ \\
\hline Caso 3 & 26 & $\mathrm{~F}$ & Cabeça pancreática & Duodenopancreatectomia \\
\hline Caso 4 & 19 & $\mathrm{~F}$ & Cabeça pancreática & Duodenopancreatectomia \\
\hline
\end{tabular}

O tamanho das lesões variou de $6,5 \mathrm{~cm}$ (caso 3 ) a $15 \mathrm{~cm}$ (caso 2). Ao exame macroscópico, o tumor se apresentava com aspecto císticopapilar, com conteúdo hemorrágico em três casos e, em apenas um, era sólido-papilar (caso 4). Em todas as peças cirúrgicas havia margens livres de tumor. A microscopia mostrou no caso 1 invasão vascular e no caso 2 invasão angio-linfática. O estudo imunoistoquímico foi realizado em três casos e é apresentado na Tabela 2 .

TABELA 2 - Imunoistoquímica dos tumores

\begin{tabular}{lccc}
\hline Paciente & Caso 1 & Caso 2 & Caso 4 \\
\hline Vimentin (V9) & + & + & + \\
Alfa-1 anti-tripsina & + & $\mathrm{NP}$ & + \\
Cromogranina A & + & $\mathrm{NP}$ & - \\
Enolase neuro específica & + & + & + \\
Receptor de progesterona & $\mathrm{NP}$ & - & $\mathrm{NP}$ \\
Proteína S100 & + & + & $\mathrm{NP}$ \\
Citoqueratina (AE1, AE3) & - & $\mathrm{NP}$ & + \\
\hline
\end{tabular}

$\mathrm{NP}=$ Antígeno não pesquisado

O tempo médio de seguimento foi de 15 meses (1 a 27 meses), estando todos os pacientes vivos até a presente data, sem evidências de recurrência ou metástases. 


\section{DISCUSSÃO}

A primeira descrição do TSPP foi feita por Frantz em 1959, quando relatou como uma nova entidade quatro casos previamente definidos como neoplasias não-funcionantes de células de ilhotas pancreáticas ${ }^{(2,16)}$. Essa neoplasia tem sido descrita sob vários termos: "papillary cystic neoplasm", "solid and cystic acinar cell tumor", "papillary cystic neoplasm", "papillary cystic carcinoma", "solid and cystic tumor" e "low-grade papillary tumor". Apenas recentemente, em 1996, esse tumor foi denominado "solidpseudopapillar tumor" pela Organização Mundial de Saúde ${ }^{(7,10)}$.

O tumor de Frantz é uma neoplasia rara do pâncreas, ocorrendo em aproximadamente $0,17 \%-2,7 \%$ dos tumores não-endócrinos do pâncreas ${ }^{(16)}$. Até o momento, existem cerca de 452 casos relatados desse tumor na literatura inglesa ${ }^{(9)}$. Recentemente, tem sido notado o aumento de sua incidência com mais de $2 / 3$ dos casos totais descritos nos últimos 10 anos. A possível explicação é o maior conhecimento da doença e a maior uniformidade de conceituação nos últimos anos ${ }^{(10)}$. No Brasil, a maior casuística em uma única instituição foi feito pelo Serviço de Cirurgia de Vias Biliares e Pâncreas do Hospital das Clínicas da Faculdade de Medicina da Universidade de São Paulo, com 14 casos operados ${ }^{(1)}$.

A origem do tumor de Frantz é, sem dúvida, grande motivo de controvérsia. A despeito do seu grande uso, o método imunoistoquímico tem se revelado de auxílio apenas marginal no esclarecimento dessa controvérsia. Resultados inconsistentes têm sido encontrados para marcadores epiteliais, neuroendócrinos, de enzimas pancreáticas, de hormônios de células de ilhotas e para outros antígenos ${ }^{(15)}$. A despeito da falta de diferenciação específica para determinada linhagem celular, o TSPP apresenta uma imunofenotipagem característica. A vimentina é consistentemente expressa, assim como marcadores para alfa-1 anti-tripsina, alfa-11 anti-quimiotripsina e enolase neuroespecífica ${ }^{(10,15)}$. Esses achados confirmam a opinião da maioria dos autores que concorda com a teoria da célula precursora do tumor ser oriunda de uma célula epitelial primitiva ${ }^{(12,16)}$. Os resultados dos exames de imunoistoquímica realizados nos pacientes desta casuística foram uniformemente compatíveis com a presença desses marcadores, com exceção do alfa-11 anti-quimiotripsina, que não foi testado.

A teoria da origem do tumor a partir de células embrionárias é defendida por KOSMAHL et al. ${ }^{(8)}$. Esses autores, estudando 59 tumores e aplicando grande número de marcadores em análises imunoistoquímicas, observaram forte reatividade para vimentina, alfa-1 anti-tripsina, enolase neuroespecífica e para receptores do progesterona. Em virtude dos achados não terem sido compatíveis com nenhuma linhagem celular específica do pâncreas e também pela presença do receptor hormonal nas amostras avaliadas, os autores sugeriram que algumas células embrionárias pudessem permanecer no pâncreas durante a embriogênese, diferenciandose posteriormente no TSPP. A predileção pelo sexo feminino encontrado nesses tumores dá suporte a essa teoria. LAM et al. ${ }^{(9)}$, no entanto, avaliando oito pacientes não encontraram em nenhum caso positividade imunoistoquímica para nenhum receptor hormonal. REBHANDL et al. (14), em revisão da literatura sobre o assunto, encontraram o receptor da progesterona em apenas 27 casos, sugerindo que os hormônios sexuais tenham papel no crescimento e não na patogênese desse tumor. No presente estudo em apenas um paciente em que foi testado o receptor de progesterona não houve positividade.

A maioria dos casos diagnosticados têm sido encontrada em mulheres jovens, especialmente em adolescentes, o que é compatível com $75 \%$ da presente casuística. Os achados clínicos são vagos e podem incluir dor abdominal leve e saciedade precoce ${ }^{(10)}$. No momento do diagnóstico, os tumores, comumente, apresentam grandes dimensões. A despeito disso é rara a invasão de estruturas vasculares ou biliares, o que torna a ressecção possível na maioria dos pacientes. Na maioria das séries o tumor acomete, preferencialmente, o corpo e cauda pancreática ${ }^{(10,13,14)}$. No presente estudo o inverso foi observado, com $75 \%$ dos casos ocorrendo na cabeça pancreática. Tal achado pode ser justificado pelo pequeno número de pacientes estudados.

A ressecção cirúrgica é o melhor tratamento para o TSPP, sendo na maioria das vezes, o único tratamento suficiente. O tipo de ressecção depende da topografia do tumor e deve objetivar a preservação das estruturas adjacentes. Duodenopancreatectomias com preservação do piloro e pancreatectomias corpocaudais com preservação do baço devem ser tentadas ${ }^{(1)}$. Em casos selecionados, a enucleação também é alternativa viável, o que foi realizada em um dos casos na presente série, sem morbidade e com margens cirúrgicas livres. As taxas de ressecabilidades são altas em virtude do tumor, ao crescer, deslocar as estruturas adjacentes ao invés de invadi-las ${ }^{(16)}$. A maioria dos estudos concorda que ressecções alargadas ou linfadenectomias não estão indicadas ${ }^{(14)}$. As metástases devem ser ressecadas, mesmo quando associadas à recidiva tumoral, o que é mais comum na população mais idosa ${ }^{(5,11)}$.

A experiência com o tratamento adjuvante é limitada aos bons resultados obtidos com a ressecção do tumor. Alguns esquemas de quimioterapia têm sido propostos, porém sem números suficientes para conclusões definitivas. A radioterapia foi relatada com sucesso em apenas um caso com invasão do hilo hepático(3).

Em virtude da longa história natural da doença e da relativa raridade, tem sido difícil estabelecer critérios histopatológicos preditivos de agressividade do tumor ${ }^{(6)}$. NISHIHARA et al. ${ }^{(12)}$, analisando 22 pacientes portadores de TF identificaram, em 3 casos com potencial maior de malignidade em virtude de metástases para gânglios, peritônio e órgãos distantes, a presença de invasão venosa, atipia nuclear e áreas de necrobiose, sugerindo que tais achados pudessem predizer doença mais avançada. A despeito disso, sobrevida em longo prazo de 7-10 anos tem sido relatada em pacientes com ressecção completa da lesão e também naqueles com doença residual, o que tem justificado se considerar o TSPP como uma neoplasia de bom prognóstico. No entanto, em aproximadamente $15 \%$ dos casos metástases são descritas $^{(4,5,6,17)}$, e até o momento cinco casos de morte por doença à distância foram relatados ${ }^{(10,16)}$. Em virtude de dois pacientes desta casuística apresentarem, à microscopia, invasão venosa, encontramse sob rigoroso seguimento anual no sentido de detecção precoce de metástases à distância. Por enquanto, não há sinais de recidiva em nenhum dos casos operados, o que, apesar do pequeno tempo de seguimento, certamente atesta o caráter menos agressivo dessas lesões quando comparadas ao adenocarcinoma pancreático. 
Costa-Neto GD, Amico EC, Costa GID. Solid-pseudopapillary tumor of the pancreas: report of four cases. Arq Gastroenterol 2004;41(3):259-62.

ABSTRACT - Background - Solid-pseudopapillary tumor of the pancreas is a rare neoplasia of pancreas, with a rate varying from $0.17 \%-2.7 \%$ of non-endocrine tumors of the pancreas. Recently there has been a steady increase in the number of solid-pseudopapillary tumors of the pancreas, with more than two thirds of the total cases described in the last 10 years. Objective - To evaluate four patients with Frantz's tumor in a Brazilian institution. Patients and Methods - Four patients with diagnosis of solid-pseudopapillary tumor were analysed in a retrospective analysis from December 2000 to February 2003 , clinically and histopathologically. Therapeutical approach and prognosis were also studied. Results - There were three females and one male with a median age of 27 years (range, 17-42). Dyspeptic symptoms and abdominal palpable mass represented the initial clinical findings. The main localization of the tumor was the pancreatic head (three of four cases). Enucleation was performed in one case, Whipple's surgery in two cases, and distal pancreatectomy in one case. Curative resection was possible in all cases confirmed by free margins. Two cases showed venous invasion histopathologically. Immunohistochemical analysis was done in three cases to confirm the diagnosis. In an average of 15-month follow-up, no recurrence has been observed. Conclusion - Our casuistic showed the preference of the tumor to the head of the pancreas, controversial to literature. However, other characteristics were similar to literature reports (clinical, histopathological, immunohistochemical and therapeutics). In addition, a longer postoperative follow-up will be necessary to affirm about prognosis.

HEADINGS - Pancreatic neoplasms. Carcinoma, papillary.

\section{REFERÊNCIAS BIBLIOGRÁFICAS}

1. Cunha JEM, Machado MCC, Penteado S, Jukemura J, Abdo EE, Bacchella T, Montagnini AL. Tratamento dos tumores císticos do pâncreas. In: Atualização em Cirurgia do Aparelho Digestivo e Coloproctologia. São Paulo: Frôntis Editorial; 2002. p.187-95.

2. Frantz VK. Tumor of the pancreas. In: Frantz VK, editor. Atlas of tumor pathology. Washington, DC: Armed Forces Institute of Pathology; 1959. p.32-3.

3. Friep P, Cooper J, Balthazar E, Fazzin E, Newall J. A role for radiotherapy in the treatment of solid and papillary neoplasms of the pancreas. Cancer 1985;56:2783-5.

4. Hernadez-Maldonado JJ, Rodriguez-Bigas MA, Gonzales DP, Vazquez-Quintana E. Papillary cystic neoplasm of the pancreas. A report of a case presenting with carcinomatosis. Am Surg 1989;55:552-9.

5. Horisawa M, Niinomi N, Sato T, Yokoi S, Oda K, Ichikawa M, Anjo SH. Frantz tumor (solid and cystic tumor of the pancreas) with liver metastasis: successful treatment and long-term follow-up. J Pediatr Surg 1995;30:724-6.

6. Kay W. Solid-pseudopapillary tumor of the pancreas: challenges presented by an unusual pancreatic neoplasm. Ann Surg Oncol 2002;9:3-4.

7. Kloppel G, Solcia E, Longnecker DS, Capella C, Sobin LH. Histological typing of tumors of the exocrine pancreas. New York: Springer; 1996.

8. Kosmahl M, Seada LS, Jänig V, Harms D, Klöppel G. Solid-pseudopapillary tumor of the pancreas: its origin revisited. Virchows Arch 2000;436:473-80.

9. Lam KY, Lo CY, Fan ST. Pancreatic solid-cystic-papillary tumor: clinicopathologic features eight patients from Hong Kong and review of the literature. World J Surg 1999;23:1045-50.

10. Martin RCG, Klimtra DS, Brennan MF, Conlon KC. Solid-pseudopapillary tumor of the pancreas: a surgical enigma? Ann Surg Oncol 2002;9:35-40.
11. Meniconi MT, Sousa MV, Rodrigues AL, Mancero JM, Quireze JC, Cardoso ES, D’Albuquerque LA, Araújo SR, Santo GC, Oliveira e Silva A. Tumor de Frantz. Relato de dois casos. Medidas terapêuticas e prognóstico. Arq Gastroenterol 1997;34:43-8.

12. Nishihara K, Nagoshi M, Trusseyoshi M, Yamaguchi K, Hayashi I. Papillary cystic tumors of the pancreas. Cancer 1993;71:82-92.

13. Pettinato G, Manivel JC, Ravetto C, Tenacciano LM, Gould EW, di Tuoro A, Jaszcz W, Albores-Saavecha A. Papillary cystic tumor of the pancreas. A clinicopathologic study of 20 cases with cytologic, immunohistochemical, ultrastructural and flow cytometric observations, and a review of the literature. Am J Clin Pathol 1992;98:478-88.

14. Rebhandl W, Felberbauer FX, Puig F, Paya K, Hochschorner S, Barlan M, Horcher E. Solid-pseudopapillary tumor of the pancreas (Frantz tumor) in children: report of four cases and review of the literature. J Surg Oncol 2001;76:289-96.

15. Solcia E, Capella C, Kloppel G. Tumors of the pancreas. In: Rosai J, Sobin LH, editors. Atlas of tumor pathology, 3rd series. Washington, DC: Armed Forces Institute of Pathology; 1997. p.31-144.

16. Yoon DY, Hines OJ, Bilchik AJ, Lewin K, Cortina G, Reber HA. Solid and papillary epithelial neoplasms of the pancreas: aggressive resection for cure. Am Surg 2001;67:1195-9.

17. Zinner MJ, Shurbaji MS, Cameron JL. Solid and papillary epithelial neoplasms of the pancreas. Surgery 1990;108:475-80. 